\title{
Identification of Atrial Natriuretic Factor within Ventricular Tissue in Hamsters and Humans with Congestive Heart Failure
}

Brooks S. Edwards, Douglas M. Ackermann, Mu E. Lee, Guy S. Reeder, Lester E. Wold, and John C. Burnett, Jr. Division of Cardiovascular Diseases, Department of Internal Medicine, Department of Pathologic Anatomy, and Department of Physiology and Biophysics, Mayo Medical School, Rochester, Minnesota 55905

\begin{abstract}
In normal mammals, atrial natriuretic factor (ANF) is present within atrial myocardial cells but is absent from ventricular myocardium. In primitive organisms ANF is present within both atria and ventricle, suggesting that the ventricle may participate both in the synthesis and release of the hormone. The current study was designed to test the hypothesis that ventricular ANF develops as a homeostatic response to intravascular volume overload. Studies were performed on cardiac tissue obtained from (i) normal and cardiomyopathic hamsters, (ii) autopsied humans with and without cardiac disease, and (iii) living humans with congestive heart failure (CHF) undergoing diagnostic right ventricular endomyocardial biopsy. The myocardium was examined for the presence of immunoreactive ANF using a two-stage immunohistochemical technique, with nonimmune rabbit sera used as a negative control. There was unequivocal evidence of focal subendocardial deposits of immunoreactive ANF present in both of the ventricles of all six cardiomyopathic hamsters, four of five autopsied human subjects with CHF, and five of seven biopsied humans. No immunoreactive ANF was observed within the ventricular myocardium of control hamsters or normal humans. Utilizing crude tissue homogenates and radioimmunoassay techniques, the quantity of ANF was determined in cardiac atria, ventricles, and noncardiac skeletal muscle. Heart failure is characterized by a reduction in atrial ANF and an increase in ventricular ANF. This study demonstrates immunoreactive ANF is present within the ventricular myocardium in cardiomyopathic hamsters and humans with $\mathrm{CHF}$, and suggests that the ventricle may be capable of responding to chronic volume overload by producing ANF.
\end{abstract}

\section{Introduction}

Atrial natriuretic factor (ANF) ${ }^{1}$ is a peptide hormone of cardiac origin. It is hypothesized that this hormone may play a fundamental role in the regulation of extracellular fluid volume and the control of systemic arterial pressure. The initial

Address reprint requests to Dr. Edwards, Dept. of Internal Medicine, Mayo Clinic and Foundation, 200 First St. S.W., Rochester, MN 55905.

Received for publication 29 September 1986 and in revised form 5 June 1987.

1. Abbreviations used in this paper: ANF, atrial natriuretic factor; $\mathrm{CHF}$, congestive heart failure.

J. Clin. Invest.

(c) The American Society for Clinical Investigation, Inc

0021-9738/88/01/0082/05 $\$ 2.00$

Volume 81 , January $1988,82-86$ studies of deBold and colleagues (1) demonstrated that extracts of atrial tissue obtained from normal animals contained a natriuretic substance. Extracts of ventricular tissue did not possess natriuretic activity. Since these landmark observations were reported, the molecular biology of ANF has been extensively examined. The peptide sequence of the hormone and its precursors have been established. The gene responsible for the production of the hormone has been identified, and the specific mRNA is fully characterized (2).

Immunohistochemical techniques have been developed to assess the presence and relative density of ANF containing secretory granules within the heart. Previous studies have utilized this technique to map ANF content within both the atria and to investigate extra cardiac sites of ANF production in the normal animal (3).

Chimoskey and co-workers (4) have reported that the atrial content of ANF is reduced in the cardiomyopathic hamster, an animal model of congestive heart failure (CHF). This observation led investigators to speculate that the sodium retention associated with CHF may result from an ANF deficiency. However, we have recently reported that there exists an inverse relationship between circulating levels of ANF and atrial content of the peptide (5). The cardiomyopathic hamster is characterized by reduced atrial ANF granularity and increased circulating levels of ANF. This situation is characteristic of a highly stimulated system in which ANF is rapidly synthesized and released, with minimal reserve storage. While the cardiomyopathic hamster is not characterized by an absolute deficiency of ANF, there may exist a relative deficiency given the marked cardiac volume overload. The human with $\mathrm{CHF}$ also exhibits a marked elevation in circulating levels of ANF. In both laboratory animals and humans with $\mathrm{CHF}$, circulating ANF is consistently increased (6). We hypothesize that severe CHF is characterized not only by cardiac volume overload with stimulation of atrial synthesis of ANF, but also by stimulation of ventricular synthesis of this important regulatory peptide.

The current study was undertaken to investigate whether ANF could be detected in ventricular tissue obtained from $(a)$ hamsters with hereditary cardiomyopathy, and $(b)$ human autopsy and biopsy material from individuals with the syndrome of CHF. Further, the quantity of ANF present within cardiac and noncardiac tissue was assessed utilizing radioimmunoassay techniques.

\section{Methods}

Immunohistochemical studies. Studies were performed in cardiomyopathic hamsters, age- and sex-matched control hamsters, and in humans with $\mathrm{CHF}$ and matched control humans.

Hamsters. Six male Syrian cardiomyopathic hamsters (Bio 14.6) and six matched control hamsters (F1B; BioBreeders Inc., Watertown, MA) were used for this study. The cardiac weight, atrial granularity, 
and plasma levels of ANF have previously been reported for the animals in this group (5). The hamsters were all $231 \mathrm{~d}$ of age at the time of the study. Each animal was anesthetized with thiobutabarbital (100 $\mathrm{mg} / \mathrm{kg}$ i.p.). Blood was collected by decapitation and the viscera were rapidly excised and fixed in 10\% buffered formalin.

The hearts were assigned random numbers and the investigators were blinded as to the group from which the hearts originated. After adequate fixation, the hearts were dissected, weighed, and visually inspected. The ventricular mass was sectioned transversely in the middle one-third of the chamber so as to orient the ventricles in a short axis configuration. The tissue was dehydrated and embedded in paraffin. Tissue sections were cut at a thickness of $5 \mu \mathrm{m}$ and mounted on glass slides. Serial sections were stained with hematoxylin and eosin to ensure proper orientation.

Autopsied humans. The presence of ventricular ANF was investigated in ten human hearts obtained at autopsy, five from individuals with clinical and pathologic evidence of chronic CHF (average age, $59 \pm 3 \mathrm{yr}$ ), and five age-matched (average age, $55 \pm 3 \mathrm{yr}$ ) control human hearts from subjects who died of noncardiac causes (trauma, two cases; lymphoma, leukemia, and breast cancer, one case each). In each case the heart was examined both grossly and microscopically.

Sections of the heart were taken from all four chambers. Atrial sections were taken from the right and left atrial appendages. Full thickness ventricular sections were obtained from the middle one-third of the left ventricular lateral free wall and the right ventricular free wall. Tissue was dehydrated and embedded in paraffin. Sections were cut to a $5 \mu \mathrm{m}$ thickness.

Right ventricular endomyocardial biopsy. Ventricular tissue was obtained sequentially from seven humans undergoing diagnostic transvenous right ventricular endomyocardial biopsy with a clinical diagnosis of CHF and/or idiopathic dilated cardiomyopathy. The average age of the subjects was $56 \pm 3$ yr and there were five males and two females studied.

Three to five specimens obtained from the right ventricular septum with a transvenous biatome were immediately fixed in $10 \%$ buffered formalin. After fixation, the tissue was dehydrated and embedded in paraffin. Serial sections were cut at a thickness of 3-5 $\mu \mathrm{m}$.

Immunohistochemical technique. The presence of ventricular ANF was assessed utilizing an immunohistochemical technique as we have previously described (5). Briefly, we have modified the immunohistochemical technique of Chapeau and associates (3) by using a two-stage process. Slides were placed in a 3\% hydrogen peroxide solution for 5 min, which was followed by $30 \mathrm{~min}$ of exposure to a suspension of egg albumin to minimize nonspecific binding. One slide from each case was exposed to a 1:800 dilution of rabbit anti- $\alpha$-ANP serum (RAS8798; Peninsula Laboratories, Belmont, CA), and a second slide was exposed to a 1:800 dilution of a nonspecific rabbit serum. Both sets of slides were incubated for $46 \mathrm{~h}$ at $4^{\circ} \mathrm{C}$ and then for $2 \mathrm{~h}$ at $22^{\circ} \mathrm{C}$. After incubation, the tissue was washed in phosphate-buffered saline and then exposed for $20 \mathrm{~min}$ at room temperature to a 1:100 dilution of goat anti-rabbit IgG covalently linked to horseradish peroxidase. The peroxidase was visualized in a medium containing $10 \mathrm{mg}$ of amino 9-ethyl carbozol dissolved in $2.5 \mathrm{ml}$ of $\mathrm{N}, \mathrm{N}$-dimethyl formamide, 47.5 $\mathrm{ml}$ acetate buffer (pH 5.2), and $0.5 \mathrm{~cm}^{3} 3 \%$ hydrogen peroxide. Slides from the hamsters were counterstained with hematoxylin to enhance nuclear detail. The presence of ANF granules was assessed by microscopic examination of the final slides. Two trained observers reviewed the sections without knowledge as to whether the tissue belonged to the heart failure group or the normal group. ANF in the ventricle was graded as present or absent.

Quantitation of tissue ANF. Studies were designed to quantitatively assess and compare immunoreactive ANF detected within the ventricular myocytes, atrial myocytes, and noncardiac skeletal muscle of hamsters with and without heart failure. 24 male cardiomyopathic hamsters (Bio 14.6), $333 \mathrm{~d}$ of age, and 12 male control hamsters of the F1 B strain, also $333 \mathrm{~d}$ of age, were used for the preparation of ventricular extracts. Crude homogenates were prepared according to the method of DeBold (1). Animals were anesthetized with thiobutabarbi- tal $(100 \mathrm{mg} / \mathrm{kg}$ ip). The heart was rapidly excised and placed in the cooled phosphate-buffered saline. Each heart was washed of excess blood, the atria was excised $2 \mathrm{~mm}$ below the atrioventricular ring, and the chambers were opened to assure adequate removal of intracavitary blood. Diaphragms were removed and served as noncardiac skeletal muscle control tissue. The tissues were weighed, placed in cold phosphate-buffered saline $(10 \mathrm{ml} / \mathrm{g})$ and homogenated. The homogenate was centrifuged for $15 \mathrm{~min}$ at $3 \mathrm{~g}$ and $4^{\circ} \mathrm{C}$. The supernatant was collected and stored at $-70^{\circ} \mathrm{C}$ until assayed. Radioimmunoassay for ANF was performed as previously described (6). Methanol extraction on a Sepac 18 cartridge was carried out before assaying.

Results of quantitative studies are expressed as mean \pm standard error. Statistical comparisons were performed using Student's group $t$ test.

\section{Results}

Hamsters. Gross inspection of all 12 hearts by two blinded observers demonstrated that 6 cases had evidence of cardiac hypertrophy and chamber dilatation. All 6 cases were later demonstrated to belong to the cardiomyopathic group of hamsters. The hearts from the cardiomyopathic animals weighed an average of $505 \pm 69 \mathrm{mg}$, while the average cardiac weight in the control animals was $385 \pm 55 \mathrm{mg}$. The circulating ANF was significantly elevated in the cardiomyopathic hamsters compared with controls $(77.7 \pm 10.8$ vs. $185.5 \pm 27.2$ $\mathrm{pg} / \mathrm{ml}, P<0.005)$.

Immunohistochemical evaluation of the ventricular myocardium demonstrated unequivocal evidence of ventricular deposits of ANF in all animals with hereditary cardiomyopathy (Fig. 1). There was no evidence of immunoreactive ventricular ANF among the healthy control hamsters. The ANF granules were present in both ventricles and located predominantly in a perinuclear and subendocardial position. The slides treated with nonimmune rabbit sera as a negative control failed to demonstrate any immunoperoxidase activity within the ventricular myocardium. In neither group was there evidence of peroxidase activity in pericardial tissue, endothelial tissue, connective tissue, or vascular smooth muscle. Both individuals who reviewed the sections agreed on the interpretation in every case.

Autopsied humans. All human subjects with congestive cardiomyopathy demonstrated biventricular dilatation and the cardiac weight was significantly increased in the group with heart failure compared with control subjects $(698 \pm 112$ vs. $334 \pm 28 \mathrm{~g}, P<0.05)$.

Immunohistochemical preparations used antisera to alpha human ANF. Both of the blinded and independent reviewers agreed that immunoreactive ANF granules were present within the right and/or left ventricular myocytes in four of five human subjects with $\mathrm{CHF}$ (Fig. 2). The fifth subject with CHF was judged by one observer to contain ANF granules while the second observer reported ANF granules as being absent. There was no evidence of ANF immunoreactive granules within vascular smooth muscle, connective tissue, or cells comprising the visceral pericardium. The reaction occurred exclusively with ventricular myocytes. In cases with $\mathrm{CHF}$ and positive immunoreactive staining, the granules were predominantly located within the subendocardium. No ANF granules were observed in any ventricular sections obtained from control individuals. Atrial sections were positive for immunoreactive ANF in all subjects in both groups, while there was no evidence of immu- 

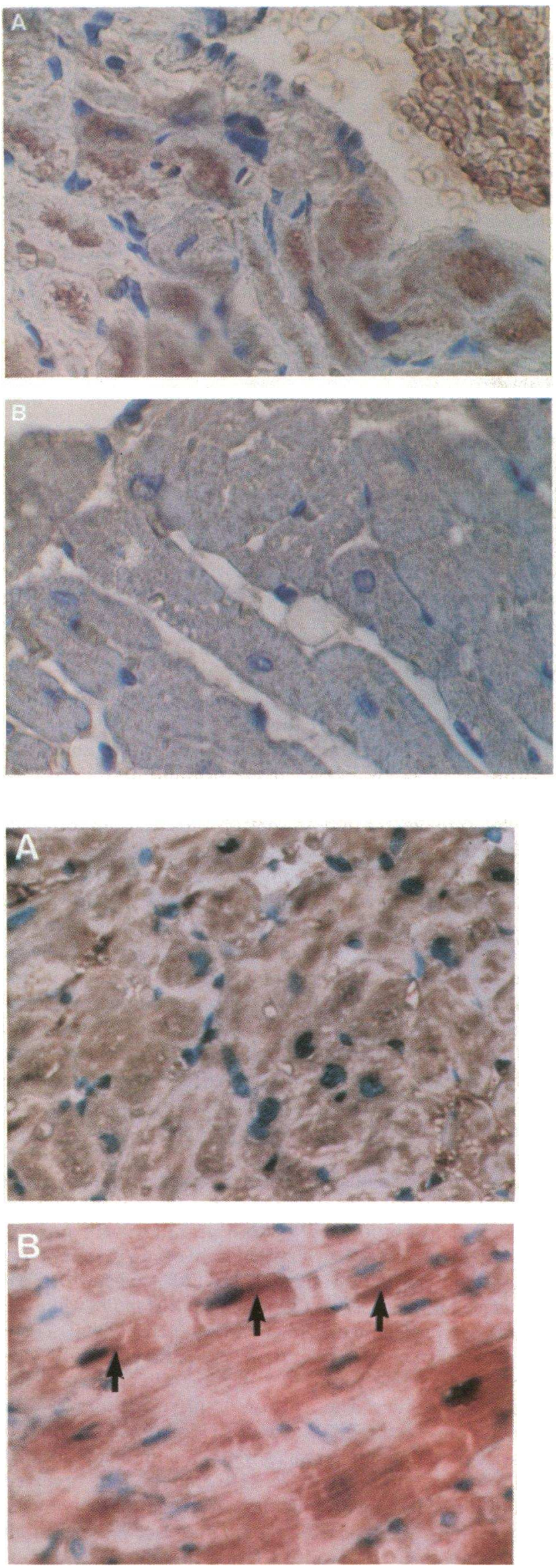
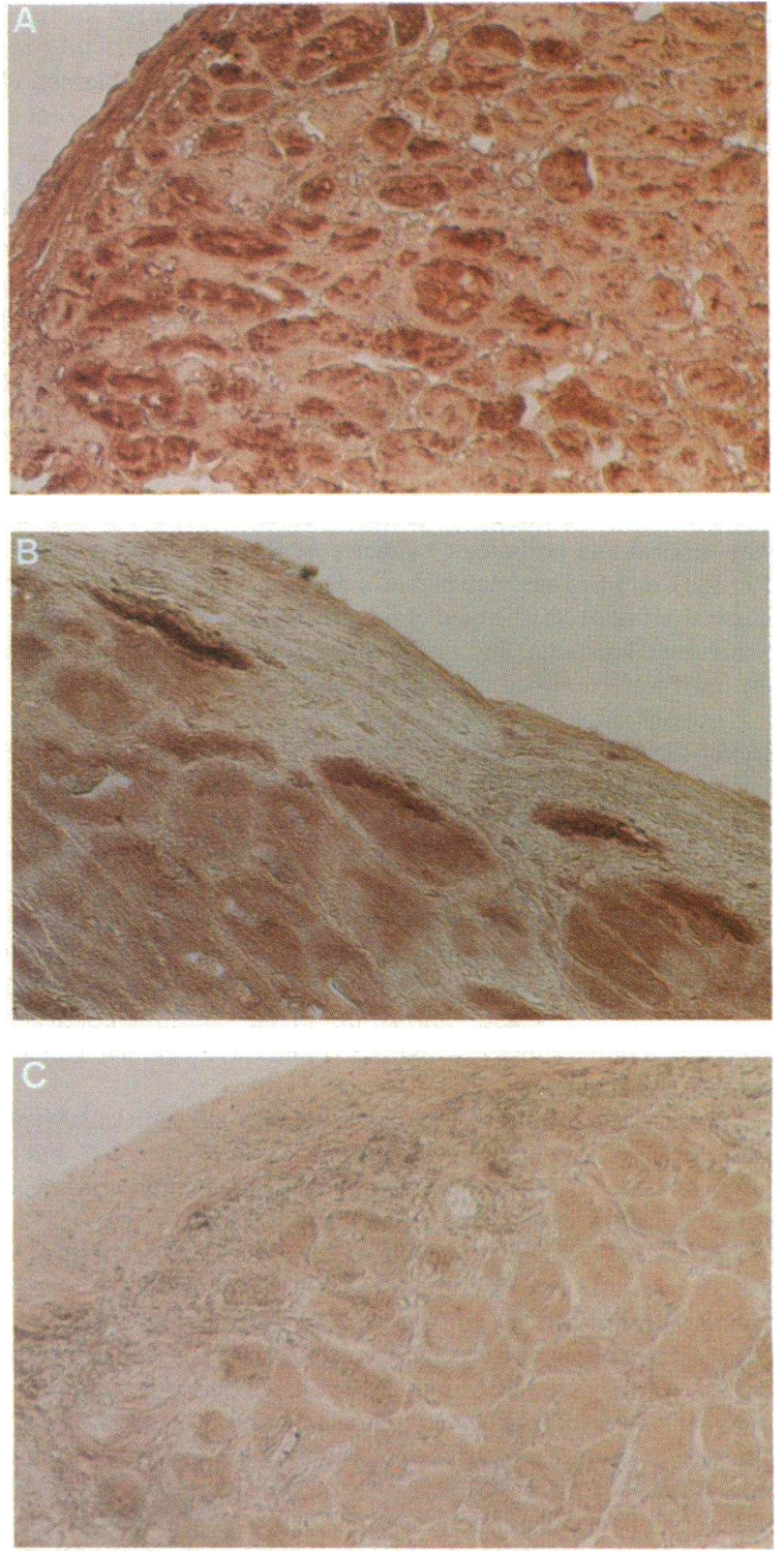

Figure 1. Immunohistochemical preparation of left ventricle from cardiomyopathic $(A)$ and normal $(B)$ hamster. Brown granules represent immunoreactive ANF. There is no evidence of immunoreactive ANF in the myocardium of the normal hamster. Nuclei counterstained with hematoxylin appear blue (original, $\times 70$ ).

Figure 2. Immunohistochemical preparation from autopsied human heart from a subject with heart failure: $(A)$ left ventricle; dense brown granules in the subendocardial region represent immunoreactive ANF (original, $\times 35) ;(B)$ high power view of left ventricular cardiocytes with immunoreactive ANF located immediately below the endocardial surface (original, $\times 70$ ); $(C)$ left ventricle treated with nonimmune rabbit serum. No evidence of specific immunoperoxidase activity (original, $\times 70$ ).

Figure 3. Immunohistochemical preparation of tissue obtained during right ventricular endomyocardial biopsy. $(A)$ Section staining negative for ANF; no perinuclear granules, only nonspecific background staining present. (B) Immunoreactive ANF granules present in a perinuclear position (arrow). Nuclei counterstained with hematoxylin. 
noreactive ANF in any section treated with nonimmune rabbit sera.

Biopsied humans. Among the seven patients studied there was clinical evidence of ventricular dysfunction in each. Left ventricular filling pressure (pulmonary capillary wedge pressure or left ventricular end-diastolic pressure) was estimated in six cases and was elevated in each (mean, $17.7 \pm 2.4 \mathrm{mmHg}$ ). In one case in which hemodynamics were not assessed invasively, the ejection fraction, as determined by echocardiography, was $28 \%$.

Among the seven cases studied, five had definite evidence of immunoreactive ANF granules with the ventricular myocytes (Fig. 3). In one patient with a clinical diagnosis of idiopathic dilated cardiomyopathy, there was no ANF observed, and in one patient with myocarditis, ANF granules were absent.

Endomyocardial biopsy was diagnostic of myocarditis in one case and suggestive of cardiac sarcoidosis in a second case. In the remaining five cases, the biopsy was nondiagnostic. In each case moderate to marked myocyte hypertrophy was present.

Tissue quantification of $A N F$. The content of immunoreactive ANF with atrium, ventricle, and diaphragm from both normal and cardiomyopathic hamsters is displayed in Fig. 4. Atrial tissue from the animals with CHF contained significantly less ANF than normal atria $(0.91 \pm 0.16$ vs. $1.69 \pm 0.03$ $\mathrm{ng} / 100 \mathrm{mg}$ wet weight, $P<0.05$ ). However, ventricular tissue from the animals with heart failure contained significantly greater quantities of ANF than that measured in normal ventricular tissue $(0.83 \pm 0.14$ vs. $0.17 \pm 0.04 \mathrm{ng} / 100 \mathrm{mg}$ wet weight, $P<0.05)$. The content of ANF within noncardiac skeletal muscle (diaphragm) did not differ between groups $(0.2 \pm 0.1$ vs. $0.2 \pm 0.07 \mathrm{ng} / 100 \mathrm{mg}$ wet weight, NS).

\section{Discussion}

These studies demonstrate the existence of immunoreactive ANF within the ventricular myocardium of animals with hereditary cardiomyopathy and CHF. These studies also importantly extend this observation to demonstrate the occurrence of ventricular ANF in the human with CHF. The absence of ANF within the normal ventricle, and the presence of ANF within the ventricle of hearts with congestive failure of various etiologies, supports the concept that heart failure is a stimulus for the production of ANF by the ventricle.

Previous studies have demonstrated the occurrence of ventricular deposits of ANF in primitive organisms (3), and recently their presence in neonatal mammalian ventricular cardiocyte has clearly been demonstrated (7). The induction of the ANF and increased quantities of ANF mRNA has been observed in ventricular tissue of rats subjected to chronic aortic constriction or dexamethasone (8). Lattion and associates have reported an increase in ANF mRNA in the ventricle of rat hearts subjected to chronic volume overload (9). The current study extends prior observations both by recognizing the presence of ANF within the ventricle of animals with chronic $\mathrm{CHF}$, and by documenting the occurrence of ventricular ANF in humans with CHF.

It is notable that in heart failure, ANF granules are found within both right and left ventricles, predominantly in a subendocardial location. It has been demonstrated, using finite ele-

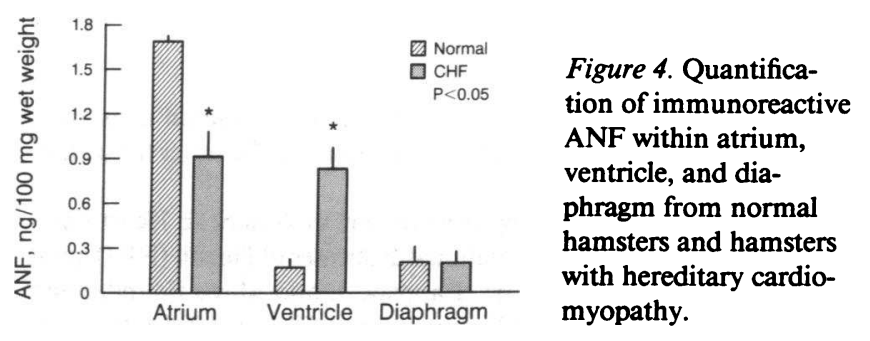

ment analysis of myocardial stress/strain relationships, that intramural tension is greatest within the subendocardial region of the ventricle (10). Increased intramural tension may represent the stimulating factor necessary for the ventricular synthesis of ANF. In addition, the subendocardial location makes it feasible that ventricular ANF may be identified in tissue obtained by endomyocardial biopsy.

Homogenates of cardiac and noncardiac tissue clearly demonstrate a significant quantity of ANF within ventricular tissue in animals with CHF. This ANF does not appear to be secondary to contamination, from elevated circulating ANF, since in the heart failure group tissue from the diaphragm also subjected to increased circulating ANF does not contain an increased quantity of ANF. Further atrial tissue in the heart failure group contains significantly less ANF than that observed in control animals. This observation is in agreement with prior studies in which atrial ANF was assessed semiquantitatively by means of immunohistochemical techniques (5). While nonspecific immunoperoxidase activity may occur at the periphery of treated tissue, the quality of staining is markedly different from the definite granules observed within myocytes. Judging by the lack of immunoreactive ANF detected within the pericardial tissue, edge artifact did not appear to significantly alter the interpretation of the tissue. The immunoperoxidase reaction is specific for the cardiac myocyte as there was no evidence of immunoreactivity in noncardiac vascular smooth muscle or connective tissue.

The significance of ventricular ANF occurring in animals and humans with $\mathrm{CHF}$ is unclear at this time. However, this observation does provide support to the interpretation that in pathophysiologic states characterized by cardiac volume overload, the ventricle as well as the atria sense and participate in volume regulation (11). The finding of ANF within the ventricle of the failing mammalian heart as well as its occurrence in more primitive ventricles suggests that ANF may represent a fundamental regulatory peptide important to both ontogeny and phylogeny. According to deBold, "the finding of secretory-like morphological characteristics in heart muscle cells in all species studied, together with the highly conserved nature of the known sequence of ANF peptides, hints at a fundamental evolutionary strategy to maintain water and electrolyte balance" (12). The observations that the ventricle appears to be capable of producing ANF in response to the stress of chronic volume overload suggests that this peptide may play a very fundamental role in the maintenance and regulation of intravascular volume.

In summary, the present studies demonstrate that ANF is present in mammalian ventricular myocardium. Further, these studies establish that the presence of ventricular ANF is associated not with normal cardiac function, but with chronic volume overload and a failing myocardium. 


\section{Acknowledgments}

The authors wish to thank June M. Hanke for secretarial assistance, and Denise Heublein, Lynn Caron, and Timothy Plummer for technical assistance.

This investigation was supported in part by American Heart Association Grant-in-Aid 86-767, National Institutes of Health (NIH) grant HL-066334, and by the Hearst, Rappaport, and Mayo Foundations. Dr. Edwards is supported by Training Grant HL07111 from the U.S. Public Health Service. Dr. Burnett is an Established Investigator of the American Heart Association.

\section{References}

1. deBold, A. J., H. B. Borenstein, A. T. Veress, and H. Sonnenberg. 1981. A rapid and potent natriuretic response to intravenous injection of atrial myocardial extract in rats. Life Sci. 28:89-94.

2. Ballerman, B. J., and B. M. Brenner. 1986. Role of atrial peptides in body fluid homeostasis. Circ. Res. 58:619-630.

3. Chapeau, C., J. Gutkowska, P. W. Schiller, R. W. Milne, G. Thibault, R. Garcia, J. Genest, and M. Cantin. 1985. Localization of immunoreactive synthetic atrial natriuretic factor (ANF) in the heart of various animal species. J. Histochem. Cytochem. 33:541-550.

4. Chimoskey, J. E., W. S. Spielman, M. A. Brandt, and S. R. Heidenan. 1984. Cardio-atria of the Bio 14.6 hamsters are deficient in natriuretic factor. Science (Wash. DC). 223:820-822.
5. Edwards, B. S., D. M. Ackermann, T. R. Schwab, D. M. Heublein, W. D. Edwards, L. E. Wold, and J. C. Burnett, Jr. 1986. The relationship between atrial granularity and circulating atrial natriuretic peptide in hamsters with congestive heart failure. Mayo Clin. Proc. 61:517-521.

6. Burnett, J. C., P. C. Kao, D. C. Hu, D. W. Heser, D. Heublein, J. P. Granger, T. J. Opgenorth, and G. S. Reeder. 1986. Atrial natriuretic peptide elevation in congestive heart failure. Science (Wash. DC). 231:1145-1147.

7. Bloch, K. D., J. G. Seidman, J. D. Naftilan, J. T. Fallon, and C. E. Seidman. 1986. Neonatal atria and ventricles secrete atrial natriuretic factor via tissue-specific secretory pathways. Cell. 47:695-702.

8. Day, M. L., D. Schwartz, R. C. Wiegand, P. T. Stockman, S. R. Brunnert, H. E. Tolunay, M. G. Currie, D. F. Standaert, and P. Needleman. 1987. Ventricular atriopeptin: unmasking of messenger RNA and peptide synthesis by hypertrophy or dexamethasone. Hypertension. 9:485-491.

9. Lattion, A.-L., J.-P. Michel, E. Arnauld, P. Corvol, and F. Soubrier. 1986. Myocardial recruitment during ANF mRNA increase with volume overload in the rat. Am. J. Physiol. 251:H890-H896.

10. Ritman, E. L., R. M. Hecthaan, R. A. Robb, and Y. C. Pao. 1978. Finite element analysis of myocardial diastolic stress and strain relationships in the intact heart. Eur. J. Cardiol. 7:SI105-SI1 19.

11. Mark, A. L. 1983. The Bezold-Jarisch reflex revisited: clinical implications of inhibitory reflexes originating in the heart. J. Am. Coll. Cardiol. 1:90-102.

12. deBold, A. J. 1985. Atrial natriuretic factor: a hormone produced by the heart. Science (Wash. DC). 230:767-770. 